\title{
An Unexpected Reaction of Isodehydracetic Acid with Amines in the Presence of 1-Ethyl-3-(3- dimethylaminopropyl) Carbodiimide Hydrochloride Yields a New Type of $\beta$-Enaminones
}

\author{
Delong Wang ${ }^{1, *(D)}$ and Hui Shi ${ }^{2}$ \\ 1 Department of Pharmaceutical Engineering, Shanxi Agricultural University, Taigu 030801, China \\ 2 State Key Laboratory of Coal Conversion, Institute of Coal Chemistry, Chinese Academy of Sciences, \\ Taiyuan 030001, China; shihui@sxicc.ac.cn \\ * Correspondence: rizhaoalong@163.com; Tel.: +86-354-6286398 \\ Academic Editor: Andrea Penoni \\ Received: 6 April 2020; Accepted: 1 May 2020; Published: 2 May 2020

\begin{abstract}
The reaction of isodehydracetic acid with amines was serendipitously found to afford $\beta$-enaminones in the presence of the coupling agent 1-ethyl-3-(3-dimethylaminopropyl) carbodiimide hydrochloride (EDC). Under the optimal reaction condition, 23 examples of $\alpha$-aminomethylene glutaconic anhydride were obtained at approximately $30-80 \%$ yields. This is a concise, operationally simple method to expediently synthesize a new type of $\beta$-enaminone-containing compound.
\end{abstract}

Keywords: isodehydracetic acid; EDC; ketene

\section{Introduction}

$\beta$-Enaminones (including $\beta$-enaminoesters) can be primely deemed as $\beta$-acyl enamines or amides interpolated by an alkene group. These compounds are intrinsically distinct from conventional enamines in reactivity and stability that readily decompose through hydrolytic or oxidative pathways $[1,2]$. $\beta$-Enaminones, however, are quite stable and easily isolated, which may be attributed to the conjugation of the enamine to a carbonyl that attenuates their reactivity. In addition to their distinct reactivity profile, $\beta$-enaminones are the important building blocks for a variety of versatile biologically active molecules like indoles [3] and pyrimidines [4], and are significant precursors for $\beta$-aminoacids [5]. Optically active $\beta$-enaminones were also used to prepare chiral ligands for diastereoselective synthesis [6]. More importantly, they have attracted attention in pharmaceutical applications, such as anticonvulsants [7-9] and $\alpha 7$ nicotinic acetylcholine receptor modulators [10]. Their stability under simulated physiological $\mathrm{pH}$ and low toxicity are exemplified by their use as orally active medicinal agents [11,12]. A combination of the above factors provides continuous impetus to develop a synthetic route to previously inaccessible or laboriously synthesized $n$-enaminone scaffolds.

Until now, numerous approaches have been developed to construct different types of $\beta$-enaminones. The classical approach is the direct condensation of amines with acyclic or cyclic 1,3-diketones or 3-ketoesters, affording the corresponding products (Figure 1a) [13-16]. When the lactams or lactims reacted with active methylene compounds, $\beta$-enaminones with an $N$-heterocycle at the $\beta$-site can be prepared (Figure 1b) $[13,14]$. Others include the methods for the construction of cyclic and $\alpha$-substituted enaminones (Figure 1c) [17-19]. These plentiful scaffolds occurring the $\beta$-enaminone unit are pretty meaningful for diversity-oriented synthesis in drug discovery that aims to efficiently generate collections of small molecules with diverse appendages, functional groups, stereochemistry and skeletons, thus yielding diverse biological activities capable of modulating a wide variety of biological process [20-22]. We herein present a full disclosure of our serendipitous findings for ready 
synthesis of a new type of $\beta$-enaminone-containing scaffold (Figure $1, \mathbf{I V}$ ) in a concise and operationally facile way.

(a) Synthesis of acyclic or cyclic $\beta$-enaminones with exocyclic nitrogen

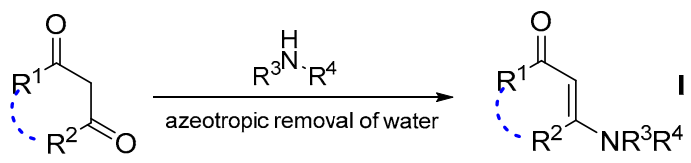

(b) Synthesis of $\beta$-enaminones with endocyclic nitrogen and exocyclic double bond
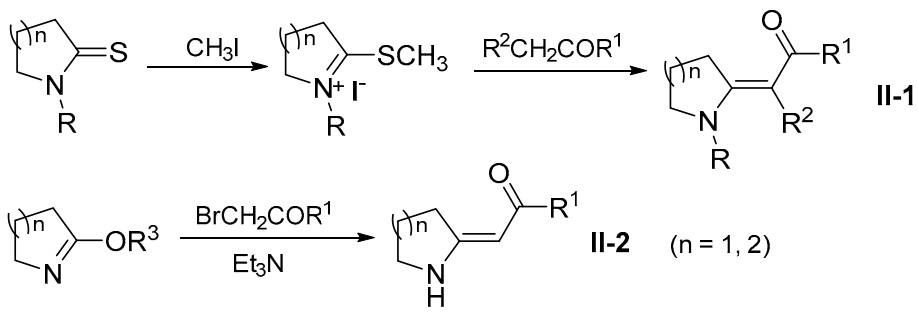

(c) Synthesis of $\beta$-enaminones with endocyclic nitrogen and endocyclic double bond

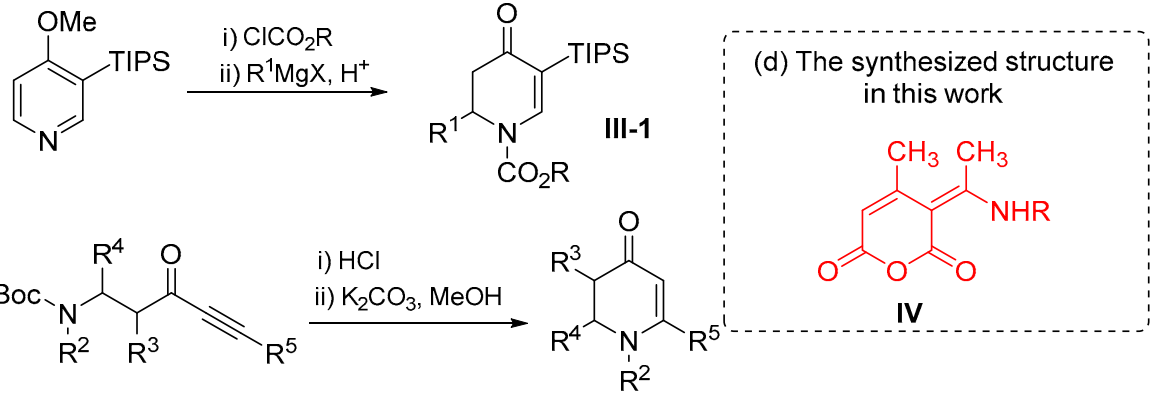

III-2

Figure 1. Synthetic pathways to different typical $\beta$-enaminones ((a) [13-16], (b) $[13,14]$, and (c) [17-19]) and the synthesized structure (IV) in present work.

\section{Results and Discussion}

Initially, we attempted to synthesize $\mathrm{N}$-propyl isodehydracetamide, a hit of succinate dehydrogenase inhibitor in our preliminary virtual screening, by simply treating isodehydracetic acid with n-propylamine in the presence of DCC/DMAP (Scheme 1). This reaction was carried out in dry DCM but resulted in a complex mixture which was different from the same reaction employing $n$-propanol instead of $n$-propylamine. Further isolation by column chromatography gave a small number of products. Analysis of its ${ }^{13} \mathrm{C}-\mathrm{NMR}$ spectrum revealed an apparent abnormal chemical shift changing from $114.5 \mathrm{ppm}$ of isodehydracetic acid to $93.4 \mathrm{ppm}$. Meanwhile, in the HMBC spectrum (Supplementary Materials), the correlations from $-\mathrm{NHC}_{2}-$ to C-6 was observed. This HMBC correlation seemed unreasonable for the anticipated compound 1 (Figure 2). These confusions had accompanied us until isobutylamine was subjected to this reaction. A cubic crystal formed from the pure product solution (50\% ethyl acetate/hexane) and the obtained X-ray structure eliminated our existing confusions. As depicted in Figure 3 (data shown in Appendix A), product 3 was a $\beta$-enaminone-containing scaffold of $\alpha$-aminomethylene glutaconic anhydride, rendering a good identity between the NMR data of compound 2 and its structure (Figure 2). 


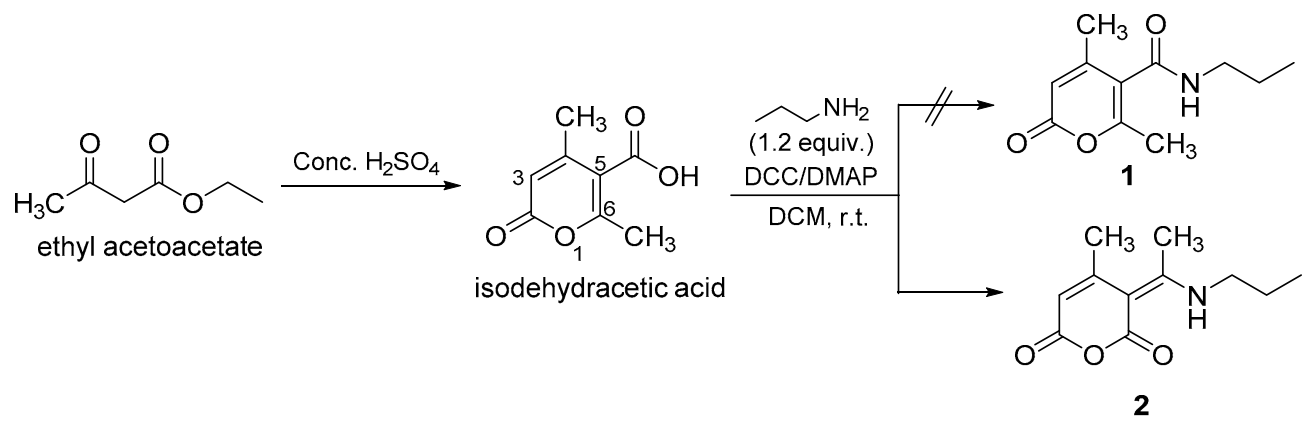

Scheme 1. Reaction of isodehydracetic acid with $n$-propylamine.
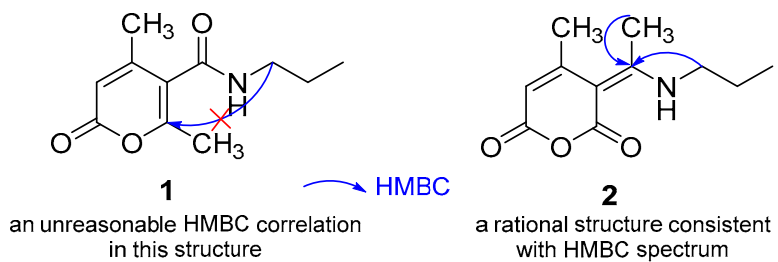

with $\mathrm{HMBC}$ spectrum

Figure 2. The observed HMBC correlations in compound $\mathbf{2}$ compared to compound $\mathbf{1}$.

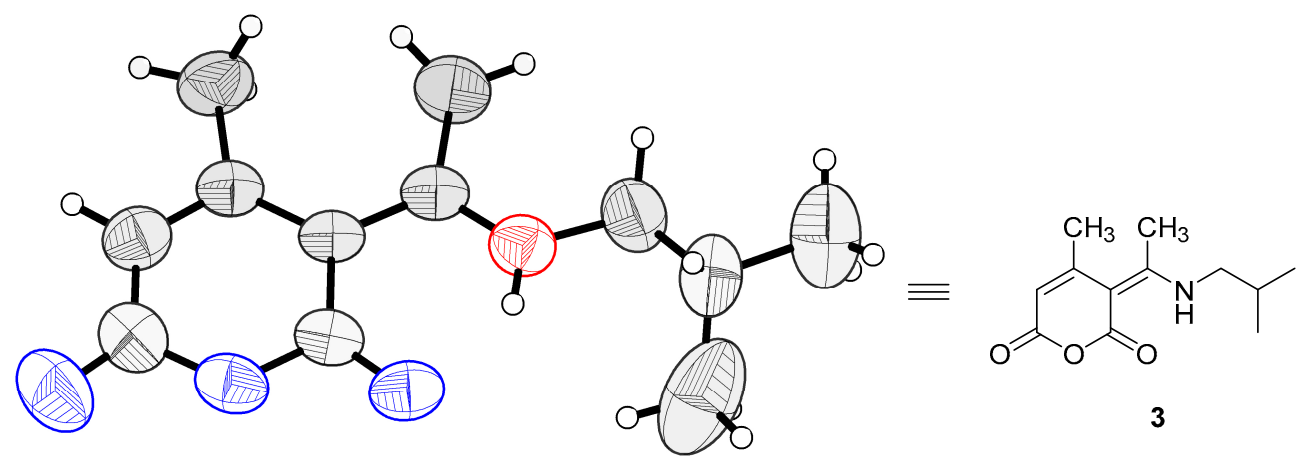

Figure 3. X-ray crystal structure of compound 3.

This reaction process was then optimized by systemically varying the reaction conditions (Table 1). As shown from entries 1-3, the EDC/HOBT reaction system was superior over DCC/DMAP and HATU/DIPEA systems. Further optimizations (entries 4 and 5) revealed that this reaction was merely relevant to EDC rather than DIPEA and $\mathrm{Et}_{3} \mathrm{~N}$. In particular, attempts to employ acyl chloride and n-propylamine failed to give a separable mixture (entry 10). Notably, decreased yields showed up when the other solvent (entry 7) or a higher temperature (entry 8) was adopted. Increasing the EDC equivalents did not improve the yield, but the reaction time could be shortened to $30 \mathrm{~min}$ (entries 4 and 6). These results disclosed that optimized conditions employed EDC for $30 \mathrm{~min}$ in DCM at room temperature.

With the optimized reaction condition established, we next explored the scope of this reaction by various amines (Scheme 2). This reaction tolerated a wide range of primary alkyl and aryl amines. As we expected, moderate yields (approximately 30\% to 80\%) were achieved. Employing alkyl amines $(2,3$, and $\mathbf{4 a}-\mathbf{4 k})$ and electron-rich phenylamines $(\mathbf{4 q}-\mathbf{4 t})$ furnished a relatively higher yield than electron-deficient phenylamines $(\mathbf{4 m}-\mathbf{4} \mathbf{p})$. Meanwhile, compared with compounds 2 and $\mathbf{4 a}$, the bulkier the chains of alkyl amines were ( 3 and $\mathbf{4 b}-\mathbf{4 e}$ ), the lower the yields of the products obtained, indicating the steric effect in the reaction process. Some exceptions to the generality of this protocol were found during substrate scope studies. As deduced from the NMR spectra, the sterically encumbered tert-butylamine and tert-octylamine gave their corresponding amide products $4 \mathbf{u}$ and $4 \mathbf{v}$, respectively, as did 2-toluidine (4w). 2,6-Dimethylaniline resulted in a complex mixture that contained neither the $\beta$-enaminone nor the amide product. 
Table 1. Optimization of reaction conditions.

\begin{tabular}{|c|c|c|}
\hline Entry & Condition & Yield of $1(\%)^{a}$ \\
\hline 1 & DCC (1.3 equiv.)/DMAP (0.1 equiv.), dry DCM, r.t., $6 \mathrm{~h}$ & 15 \\
\hline 2 & EDC (1.3 equiv.)/HOBT (1.3 equiv.), DCM, r.t., $6 \mathrm{~h}$ & 61 \\
\hline 3 & HATU (1.3 equiv.)/DIPEA (3.0 equiv.), dry DCM, r.t., $6 \mathrm{~h}$ & 36 \\
\hline 4 & EDC (1.3 equiv.), DCM, r.t., 2 h & 66 \\
\hline 5 & EDC (1.3 equiv.), DCM, r.t., $30 \mathrm{~min}$ & 62 \\
\hline 6 & EDC ( 3 equiv.), DCM, r.t., $30 \mathrm{~min}$ & 65 \\
\hline 7 & EDC (1.3 equiv.), DMF, r.t., $30 \mathrm{~min}$ & 34 \\
\hline 8 & EDC (3 equiv.), DCM, reflux, $30 \mathrm{~min}$ & n.d. b \\
\hline 9 & $\mathrm{Et}_{3} \mathrm{~N}$ (or DIPEA, DBU), $0^{\circ} \mathrm{C}$ to reflux & n.d. c \\
\hline 10 & i) $\mathrm{SOCl}_{2} ;$ ii) $\mathrm{Et}_{3} \mathrm{~N}$, THF, $0{ }^{\circ} \mathrm{C}$ to r.t. & n.d. $b$ \\
\hline
\end{tabular}

${ }^{a}$ Isolated yield. ${ }^{b}$ Inseparable mixture. ${ }^{c}$ No reaction.

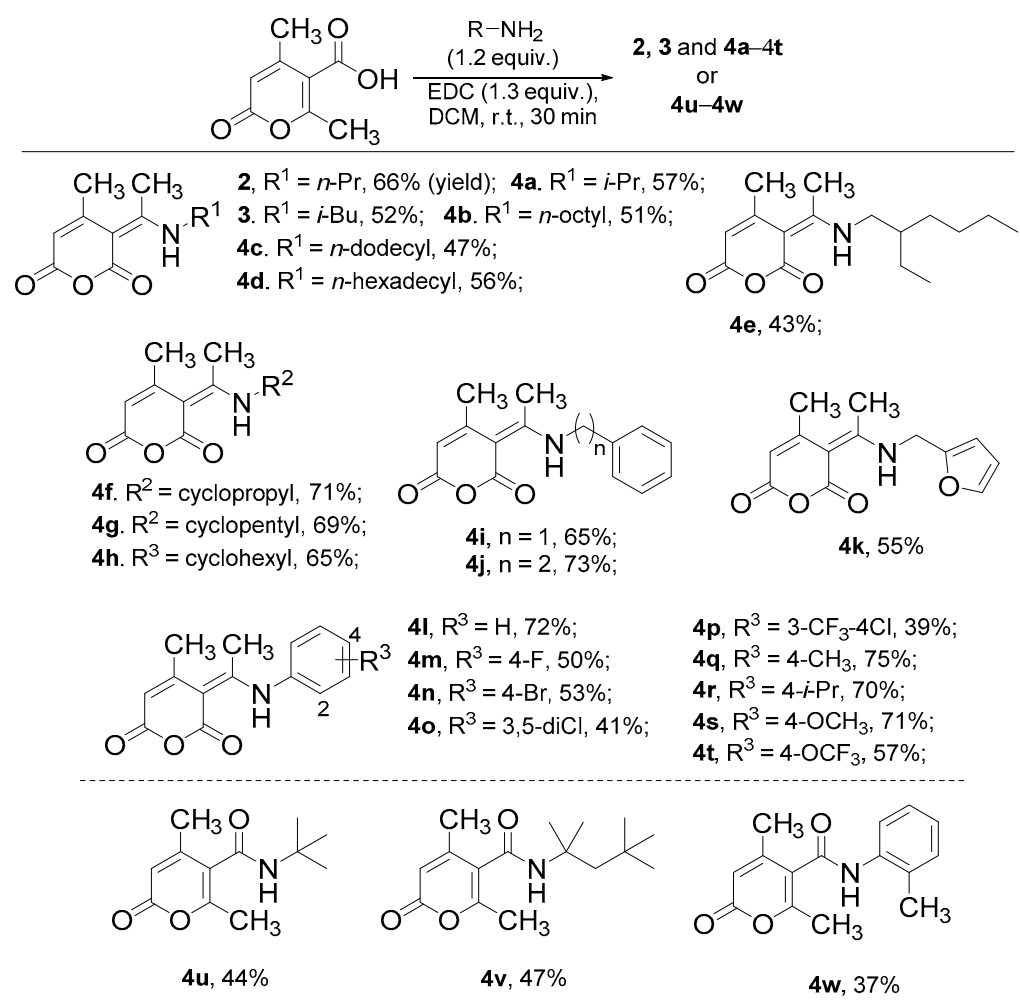

Scheme 2. Substrate scope of isodehydracetic acid.

A tentative mechanistic model of the foregoing reaction was formulated on the basis of the following considerations. It has been demonstrated that the presence of a strongly electron-withdrawing group in the $\alpha$-position to the carboxyl can enable a $\beta$-elimination pathway through an E1cB mechanism to afford the corresponding ketene [23-25]. These reaction conditions depend closely on the $\alpha$-substituent and the leaving group in E1cB elimination [24,26], and, obviously, the ketene formation under mild conditions is highly practical. Specifically, the adduct of diethylphosphonoacetic acid and DCC can readily form a ketene intermediate under mild conditions through the E1cB pathway [24]. This ketene serves as an efficient acylation agent for sterically hindered substrates in several minutes. In our case, isodehydracetic acid bears an electron-withdrawing group $\left(-\mathrm{C}\left(\mathrm{CH}_{3}\right)=\mathrm{CHCOO}-\right)$, and, therefore, when 
it reacts with EDC, a carbodiimide type of dehydrant similar to DCC (a ketene intermediate, 6) was presumptively formed first (Scheme 3). The electrophilic character of position 6 in particular would be enhanced by the ketene group at position 5, which is thus responsible for the facile acceptance of amine attack and easy elimination of the carboxyl group. This could be verified by a quantum calculation that showed a more positive charge of carbon 6 than that of the ketene carbon (Supplementary Materials). Finally, the leaving carboxyl group would be acylated by ketene, delivering the target molecule 10. However, the ketene carbon was the preference of a sterically hindered amine, thus giving the amide product 7 , as could be seen from the products $4 \mathbf{u}-4 \mathbf{w}$.

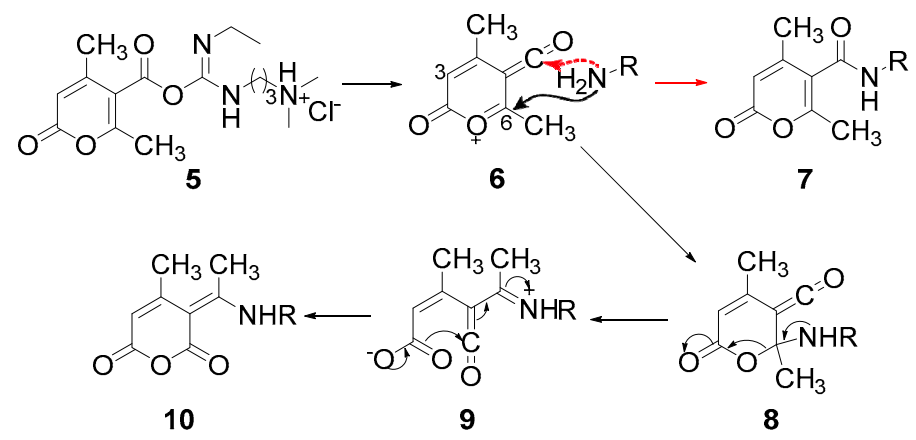

Scheme 3. Plausible mechanism for reaction of isodehydracetic acid with amines.

\section{Experimental Procedures}

\subsection{General Information}

All chemicals were purchased from Macklin Biochemical Co., Ltd. (Shanghai, China) and used without further purification. Anhydrous dichloromethane (DCM) was dispensed from a solvent purification system utilizing calcium hydride. Analytical TLC was performed using precoated plates (silica gel GF254) and visualized with UV light or an $\mathrm{I}_{2}$ chamber. Chromatography process was performed on silica gel (200-300 mesh) (Qingdao Haiyang Chemical Co., Ltd., China). A Bruker AM-500 NMR spectrometer (Bruker, Germany) was used for 1D and 2D NMR tests and the chemical shifts ( $\delta$ expressed in $p p m$ were refered to the solvent (DMSO- $\left.d_{6}\right)$ residual peaks $\left(\delta_{\mathrm{H}} 2.54 / \delta_{\mathrm{C}} 40.45\right)$. Multiplicities are given as s (singlet), d (doublet), t (triplet), q (quartet), and $\mathrm{m}$ (multiplet). Coupling constants $(J)$ are given in Hz. The HRESIMS data were collected on Waters Xevo G2-XS TOF mass spectrometer (Waters Co., Milford, MA, USA). Uncorrected melting point values were gathered using WRS-1B Digital Melting-point Apparatus (Shanghai JiaHang Instruments).

\subsection{Synthesis of Isodehydracetic Acid}

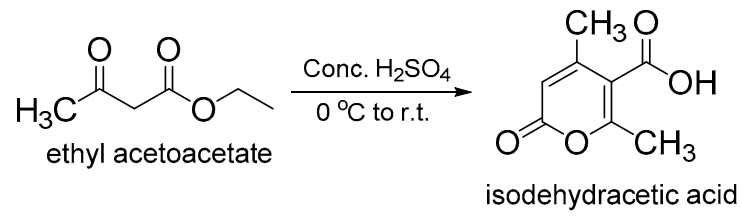

To a $2 \mathrm{~L}$ three-necked flask fitted with a thermometer, a stirrer, and a dropping funnel, $450 \mathrm{~mL}$ of concentrated sulfuric acid was added. This flask was cooled in an ice bath for $10 \mathrm{~min}$, and $325 \mathrm{~g}$ ( 2.5 moles) ethyl acetoacetate was added to the above stirring acid at such a rate that the temperature remains between $10{ }^{\circ} \mathrm{C}$ and $15^{\circ} \mathrm{C}$. When all the ester had been added, the flask was stoppered with a calcium chloride drying tube and allowed to stand at $20^{\circ} \mathrm{C}$. After 4 days, the reaction mixture was poured onto $1000 \mathrm{~g}$ of crushed ice while being stirred vigorously with a wooden paddle. The resulting solid was collected on a large Büchner funnel, washed with two $200 \mathrm{~mL}$ portions of cold water, and sucked as dry as possible. The sucked solid was then crushed and naturally air dried for a week. The 
dried solid was subjected to a silica gel column eluting with petroleum ether/ethyl acetate (1:5) to afford crude isodehydracetic acid. The crude product was dissolved in hot ethyl acetate and cooled slowly to effect crystallization. The yield of isodehydroacetic acid was approximately $30 \mathrm{~g}$, white solid,

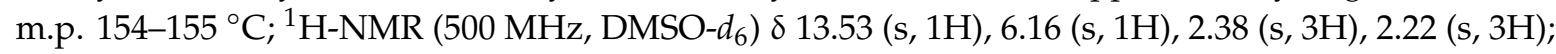
${ }^{13} \mathrm{C}-\mathrm{NMR}\left(125 \mathrm{MHz}, \mathrm{DMSO}-d_{6}\right) \delta 167.4,164.3,160.8,155.6,114.5,112.0,21.4,20.0$. ESI-MS $m / z: 169.2$ $[\mathrm{M}+\mathrm{H}]^{+}[27]$.

\subsection{General Procedure for Synthesis of Compounds $\mathbf{2}, \mathbf{3}$ and $\mathbf{4 a}-\mathbf{4} \mathbf{w}$}

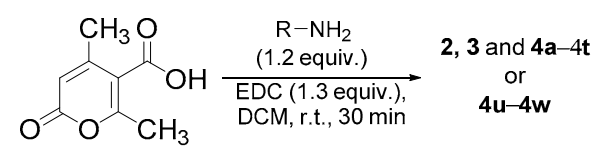

A round-bottomed flask was successively charged with isodehydracetic acid $(1.0 \mathrm{mmol})$, 1-(3-dimethylaminopropyl)-3-ethylcarbodiimide hydrochloride (EDC) (1.2 mmol) and DCM (5 mL). The mixture was stirred at room temperature for $10 \mathrm{~min}$. After that, amine $(1.2 \mathrm{mmol})$ was slowly added. The system was reacted for $30 \mathrm{~min}$. Once complete, the mixture was washed with $3 \times 5$ $\mathrm{mL}$ distilled water and the organic phase was dried with anhydrous $\mathrm{Na}_{2} \mathrm{SO}_{4}$ and concentrated. The residual was subjected to a silica gel column eluting with petroleum ether/ethyl acetate (from 5:0 to 5:1, 5:3, and 5:5) to afford a product. The product was recrystallized from petroleum ether/ethyl acetate if necessary.

(Z)-4-Methyl-3-(1-(propylamino)ethylidene)-2H-pyran-2,6(3H)-dione (2): colorless crystal, m.p. $56-58{ }^{\circ} \mathrm{C}$, yield $66 \%$; ${ }^{1} \mathrm{H}-\mathrm{NMR}\left(500 \mathrm{MHz}\right.$, DMSO- $\left.d_{6}\right) \delta 11.74(\mathrm{~s}, 1 \mathrm{H}), 5.44(\mathrm{~s}, 1 \mathrm{H}), 3.51-3.53(\mathrm{~m}, 2 \mathrm{H}), 2.51(\mathrm{~s}, 3 \mathrm{H})$, $2.35(\mathrm{~s}, 3 \mathrm{H}), 1.66-1.70(\mathrm{~m}, 2 \mathrm{H}), 0.99(\mathrm{t}, J=7.2 \mathrm{~Hz}, 3 \mathrm{H}) ;{ }^{13} \mathrm{C}-\mathrm{NMR}\left(125 \mathrm{MHz}, \mathrm{DMSO}-d_{6}\right) \delta 172.2,166.7$, 161.3, 158.9, 103.8, 94.8, 46.5, 25.5, 22.8, 20.0, 11.9. HRMS (ESI) calcd. for $\mathrm{C}_{11} \mathrm{H}_{16} \mathrm{NO}_{3}[\mathrm{M}+\mathrm{H}]^{+} 210.1125$, found 210.1129 .

(Z)-3-(1-(Isopropylamino)ethylidene)-4-methyl-2H-pyran-2,6(3H)-dione (4a): colorless crystal, m.p. $62-64{ }^{\circ} \mathrm{C}$, yield 57\%; ${ }^{1} \mathrm{H}-\mathrm{NMR}\left(500 \mathrm{MHz}, \mathrm{DMSO}-d_{6}\right) \delta 11.70(\mathrm{~s}, 1 \mathrm{H}), 5.41(\mathrm{~s}, 1 \mathrm{H}), 4.09-4.17(\mathrm{~m}, 1 \mathrm{H}), 2.51(\mathrm{~s}, 3 \mathrm{H})$, $2.31(\mathrm{~s}, 3 \mathrm{H}), 1.27(\mathrm{~s}, 3 \mathrm{H}), 1.26(\mathrm{~s}, 3 \mathrm{H}) ;{ }^{13} \mathrm{C}-\mathrm{NMR}\left(125 \mathrm{MHz}, \mathrm{DMSO}-d_{6}\right) \delta 171.3,166.9,161.5,159.2,103.8$, 94.7, 47.1, 25.6, 23.5, 20.0. HRMS (ESI) calcd. for $\mathrm{C}_{11} \mathrm{H}_{16} \mathrm{NO}_{3}[\mathrm{M}+\mathrm{H}]^{+} 210.1125$, found 210.1129.

(Z)-3-(1-(Isobutylamino)ethylidene)-4-methyl-2H-pyran-2,6(3H)-dione (3): colorless crystal, m.p. $74-76{ }^{\circ} \mathrm{C}$, yield $52 \% ;{ }^{1} \mathrm{H}-\mathrm{NMR}\left(500 \mathrm{MHz}\right.$, DMSO- $\left.d_{6}\right) \delta 11.86(\mathrm{~s}, 1 \mathrm{H}), 5.45(\mathrm{~s}, 1 \mathrm{H}), 3.39-3.42(\mathrm{~m}, 2 \mathrm{H}), 2.50(\mathrm{~s}, 3 \mathrm{H})$, $2.35(\mathrm{~s}, 3 \mathrm{H}), 1.90-2.00(\mathrm{~m}, 1 \mathrm{H}), 1.00(\mathrm{~d}, J=6.7 \mathrm{~Hz}, 6 \mathrm{H}) ;{ }^{13} \mathrm{C}-\mathrm{NMR}\left(125 \mathrm{MHz}, \mathrm{DMSO}-d_{6}\right) \delta 172.4,167.1$, 161.4, 159.1, 104.0, 94.9, 52.1, 28.8, 25.7, 20.7, 20.2. HRMS (ESI) calcd. for $\mathrm{C}_{12} \mathrm{H}_{18} \mathrm{NO}_{3}[\mathrm{M}+\mathrm{H}]^{+}$224.1281, found 224.1285 .

(Z)-4-Methyl-3-(1-(octylamino)ethylidene)-2H-pyran-2,6(3H)-dione (4b): white solid, m.p. $110-112{ }^{\circ} \mathrm{C}$, yield $51 \% ;{ }^{1} \mathrm{H}-\mathrm{NMR}\left(500 \mathrm{MHz}, \mathrm{DMSO}-d_{6}\right) \delta 11.72(\mathrm{~s}, 1 \mathrm{H}), 5.44(\mathrm{~s}, 1 \mathrm{H}), 3.52-3.54(\mathrm{~m}, 2 \mathrm{H}), 2.50(\mathrm{~s}$, $3 \mathrm{H}), 2.34(\mathrm{~s}, 3 \mathrm{H}), 1.61-1.68(\mathrm{~m}, 2 \mathrm{H}), 1.29-1.38(\mathrm{~m}, 10 \mathrm{H}), 0.89(\mathrm{t}, J=7.1 \mathrm{~Hz}, 3 \mathrm{H}) ;{ }^{13} \mathrm{C}-\mathrm{NMR}(125 \mathrm{MHz}$, DMSO- $\left.d_{6}\right) \delta 172.4,166.9,161.5,159.1,103.8,94.9,45.0,32.2,29.5,29.5,29.4,27.1,25.6,23.1,20.1,15.0$. HRMS (ESI) calcd. for $\mathrm{C}_{16} \mathrm{H}_{26} \mathrm{NO}_{3}[\mathrm{M}+\mathrm{H}]^{+}$280.1907, found 280.1912 .

(Z)-3-(1-(Dodecylamino)ethylidene)-4-methyl-2H-pyran-2,6(3H)-dione (4c): white solid, m.p. 97-99 ${ }^{\circ} \mathrm{C}$, yield $47 \%$; ${ }^{1} \mathrm{H}-\mathrm{NMR}\left(500 \mathrm{MHz}, \mathrm{DMSO}-d_{6}\right) \delta 11.72(\mathrm{~s}, 1 \mathrm{H}), 5.44(\mathrm{~s}, 1 \mathrm{H}), 3.50-3.54(\mathrm{~m}, 2 \mathrm{H}), 2.50(\mathrm{~s}$, $3 \mathrm{H}), 2.34(\mathrm{~s}, 3 \mathrm{H}), 1.60-1.67(\mathrm{~m}, 2 \mathrm{H}), 1.21-1.37(\mathrm{~m}, 18 \mathrm{H}), 0.88(\mathrm{t}, J=7.0 \mathrm{~Hz}, 3 \mathrm{H}) ;{ }^{13} \mathrm{C}-\mathrm{NMR}(125 \mathrm{MHz}$, DMSO- $\left.d_{6}\right) \delta 172.3,166.9,161.5,159.1,103.8,94.9,45.0,32.3,30.0,29.9,29.9,29.7,29.5,29.4,27.1,25.6$, 23.1, 20.1, 15.0. HRMS (ESI) calcd. for $\mathrm{C}_{20} \mathrm{H}_{34} \mathrm{NO}_{3}[\mathrm{M}+\mathrm{H}]^{+} 36.2533$, found 36.2539. 
(Z)-3-(1-(Hexadecylamino)ethylidene)-4-methyl-2H-pyran-2,6(3H)-dione (4d): white solid, m.p. $109-112{ }^{\circ} \mathrm{C}$, yield 56\%; ${ }^{1} \mathrm{H}-\mathrm{NMR}\left(500 \mathrm{MHz}, \mathrm{DMSO}-d_{6}\right) \delta 11.72(\mathrm{~s}, 1 \mathrm{H}), 5.44(\mathrm{~s}, 1 \mathrm{H}), 3.51-3.54(\mathrm{~m}, 2 \mathrm{H}), 2.51(\mathrm{~s}$, 3H), 2.35 (s, 3H), 1.63-1.69 (m, 2H), 1.21-1.37 (m, 26H), 0.89 (t, J = 7.0 Hz, 3H); ${ }^{13} \mathrm{C}-\mathrm{NMR}(125 \mathrm{MHz}$, DMSO- $\left.d_{6}\right) \delta 171.6,166.4,160.8,158.3,103.5,94.4,44.5,31.7,29.5,29.4,29.4,29.3,29.1,29.0,28.9,26.6$, 25.0, 22.5, 19.5, 14.3. HRMS (ESI) calcd. for $\mathrm{C}_{24} \mathrm{H}_{42} \mathrm{NO}_{3}[\mathrm{M}+\mathrm{H}]^{+} 392.3159$, found 392.3165 .

(Z)-3-(1-((2-Ethylhexyl)amino)ethylidene)-4-methyl-2H-pyran-2,6(3H)-dione (4e): colorless crystal, m.p. 95-96 ${ }^{\circ} \mathrm{C}$, yield 43\%; ${ }^{1} \mathrm{H}-\mathrm{NMR}\left(500 \mathrm{MHz}, \mathrm{DMSO}-d_{6}\right) \delta 11.87(\mathrm{~s}, 1 \mathrm{H}), 5.45(\mathrm{~s}, 1 \mathrm{H}), 3.48-3.50(\mathrm{~m}, 2 \mathrm{H}), 2.52$ $(\mathrm{s}, 3 \mathrm{H}), 2.36(\mathrm{~s}, 3 \mathrm{H}), 1.63-1.66(\mathrm{~m}, 1 \mathrm{H}), 1.31-1.43(\mathrm{~m}, 8 \mathrm{H}), 0.91-0.993$ (overlap, 6H); ${ }^{13} \mathrm{C}-\mathrm{NMR}(125 \mathrm{MHz}$, DMSO-d $)_{6} \delta 172.4,167.1,161.2,158.9,103.9,94.9,47.8,39.3,31.2,29.0,25.6,24.6,23.3,20.1,14.8,11.5$. HRMS (ESI) calcd. for $\mathrm{C}_{16} \mathrm{H}_{26} \mathrm{NO}_{3}[\mathrm{M}+\mathrm{H}]^{+}$280.1907, found 280.1914.

(Z)-3-(1-(Cyclopropylamino)ethylidene)-4-methyl-2H-pyran-2,6(3H)-dione (4f): white solid, m.p. $77-79{ }^{\circ} \mathrm{C}$, yield 71\%; ${ }^{1} \mathrm{H}-\mathrm{NMR}\left(500 \mathrm{MHz}, \mathrm{DMSO}-d_{6}\right) \delta 11.64(\mathrm{~s}, 1 \mathrm{H}), 5.48(\mathrm{~s}, 1 \mathrm{H}), 3.03-3.06(\mathrm{~m}, 1 \mathrm{H}), 2.67(\mathrm{~s}, 3 \mathrm{H})$, $2.36(\mathrm{~s}, 3 \mathrm{H}), 0.97-1.01(\mathrm{~m}, 2 \mathrm{H}), 0.80-0.84(\mathrm{~m}, 2 \mathrm{H}) ;{ }^{13} \mathrm{C}-\mathrm{NMR}\left(125 \mathrm{MHz}, \mathrm{DMSO}-d_{6}\right) \delta 174.5,166.7,161.1$, $158.8,104.4,94.9,27.2,25.5,21.0,8.4$. HRMS (ESI) calcd. for $\mathrm{C}_{11} \mathrm{H}_{14} \mathrm{NO}_{3}[\mathrm{M}+\mathrm{H}]^{+} 208.0968$, found 208.0974.

(Z)-3-(1-(Cyclopentylamino)ethylidene)-4-methyl-2H-pyran-2,6(3H)-dione (4g): white solid, m.p. 105-107 ${ }^{\circ} \mathrm{C}$, yield 69\%; ${ }^{1} \mathrm{H}-\mathrm{NMR}\left(500 \mathrm{MHz}, \mathrm{DMSO}-d_{6}\right) \delta 11.91(\mathrm{~s}, 1 \mathrm{H}), 5.45$ (s, 1H), 4.31-4.33 (m, $1 \mathrm{H}), 2.55(\mathrm{~s}, 3 \mathrm{H}), 2.35(\mathrm{~s}, 3 \mathrm{H}), 2.02-2.08(\mathrm{~m}, 2 \mathrm{H}), 1.61-1.75(\mathrm{~m}, 6 \mathrm{H}) ;{ }^{13} \mathrm{C}-\mathrm{NMR}\left(125 \mathrm{MHz}, \mathrm{DMSO}-d_{6}\right) \delta$ $171.5,166.9,161.3,159.0,103.8,94.8,56.3,33.9,25.5,24.4,20.5$. HRMS (ESI) calcd. for $\mathrm{C}_{13} \mathrm{H}_{18} \mathrm{NO}_{3}[\mathrm{M}+$ $\mathrm{H}]^{+}$236.1281, found 236.1289 .

(Z)-3-(1-(Cyclohexylamino)ethylidene)-4-methyl-2H-pyran-2,6(3H)-dione (4h): white solid, m.p. $122-124^{\circ} \mathrm{C}$, yield 65\%; ${ }^{1} \mathrm{H}-\mathrm{NMR}\left(500 \mathrm{MHz}, \mathrm{DMSO}-d_{6}\right) \delta 11.90(\mathrm{~s}, 1 \mathrm{H}), 5.45(\mathrm{~s}, 1 \mathrm{H}), 3.87-3.93(\mathrm{~m}, 1 \mathrm{H}), 2.55(\mathrm{~s}, 3 \mathrm{H})$, 2.36 (s, 3H), 1.31-1.92 (m, 10H); ${ }^{13} \mathrm{C}-\mathrm{NMR}\left(125 \mathrm{MHz}, \mathrm{DMSO}-d_{6}\right) \delta 171.0,166.9,161.2,159.0,103.9,94.7$, 53.2, 33.0, 25.6, 25.5, 24.4, 19.8. HRMS (ESI) calcd. for $\mathrm{C}_{14} \mathrm{H}_{20} \mathrm{NO}_{3}[\mathrm{M}+\mathrm{H}]^{+}$250.1438, found 250.1443.

(Z)-3-(1-(Benzylamino)ethylidene)-4-methyl-2H-pyran-2,6(3H)-dione (4i): white solid, yield 65\%; ${ }^{1} \mathrm{H}-\mathrm{NMR}$ (500 MHz, DMSO-d $) \delta 11.99(\mathrm{~s}, 1 \mathrm{H}), 7.35-7.46(\mathrm{~m}, 5 \mathrm{H}), 5.48(\mathrm{~s}, 1 \mathrm{H}), 4.83(\mathrm{~s}, 2 \mathrm{H}), 2.52(\mathrm{~s}, 3 \mathrm{H}), 2.35(\mathrm{~s}$, $3 \mathrm{H}) ;{ }^{13} \mathrm{C}-\mathrm{NMR}\left(125 \mathrm{MHz}, \mathrm{DMSO}-d_{6}\right) \delta 172.2,166.7,161.2,158.9,137.3,129.8,128.7,128.4,104.4,95.2$, 48.4, 25.6, 20.3. HRMS (ESI) calcd. for $\mathrm{C}_{15} \mathrm{H}_{16} \mathrm{NO}_{3}[\mathrm{M}+\mathrm{H}]^{+} 258.1125$, found 258.1129 .

(Z)-4-Methyl-3-(1-(phenethylamino)ethylidene)-2H-pyran-2,6(3H)-dione (4j): white solid, yield 73\%; ${ }^{1} \mathrm{H}-\mathrm{NMR}\left(500 \mathrm{MHz}, \mathrm{DMSO}-d_{6}\right) \delta 11.72(\mathrm{~s}, 1 \mathrm{H}), 7.26-7.37(\mathrm{~m}, 5 \mathrm{H}), 5.44(\mathrm{~s}, 1 \mathrm{H}), 3.82(\mathrm{t}, \mathrm{J}=7.0$ $\mathrm{Hz}, 2 \mathrm{H}), 2.98(\mathrm{t}, J=7.0 \mathrm{~Hz}, 2 \mathrm{H}), 2.43(\mathrm{~s}, 3 \mathrm{H}), 2.30(\mathrm{~s}, 3 \mathrm{H}) ;{ }^{13} \mathrm{C}-\mathrm{NMR}\left(125 \mathrm{MHz}, \mathrm{DMSO}-d_{6}\right) \delta 172.1,166.6$, 161.3, 158.8, 138.9 129.8, 129.4, 127.5, 104.0, 94.9, 46.3, 35.5, 25.5, 20.0. HRMS (ESI) calcd. for $\mathrm{C}_{16} \mathrm{H}_{18} \mathrm{NO}_{3}$ $[\mathrm{M}+\mathrm{H}]^{+}$272.1281, found 272.1281 .

(Z)-3-(1-((Furan-2-ylmethyl)amino)ethylidene)-4-methyl-2H-pyran-2,6(3H)-dione (4k): white solid, yield 55\%; ${ }^{1} \mathrm{H}-\mathrm{NMR}\left(500 \mathrm{MHz}, \mathrm{DMSO}-d_{6}\right) \delta 11.94(\mathrm{~s}, 1 \mathrm{H}), 7.73(\mathrm{~s}, 1 \mathrm{H}), 6.51(\mathrm{~m}, 2 \mathrm{H}), 5.55(\mathrm{~s}, 1 \mathrm{H}), 4.87$ (d, $J=5.0 \mathrm{~Hz}, 2 \mathrm{H}), 3.82(\mathrm{t}, J=7.0 \mathrm{~Hz}, 2 \mathrm{H}), 2.98(\mathrm{t}, J=7.0 \mathrm{~Hz}, 2 \mathrm{H}), 2.60(\mathrm{~s}, 3 \mathrm{H}), 2.36(\mathrm{~s}, 3 \mathrm{H}) ;{ }^{13} \mathrm{C}-\mathrm{NMR}(125$ MHz, DMSO- $\left.d_{6}\right) \delta 172.2,166.8,161.1,158.9,150.1,144.4,111.7,109.6,104.7,95.2,41.6,25.6,20.1$. HRMS (ESI) calcd. for $\mathrm{C}_{13} \mathrm{H}_{14} \mathrm{NO}_{4}[\mathrm{M}+\mathrm{H}]^{+} 248.0917$, found 248.0921 .

(Z)-4-Methyl-3-(1-(phenylamino)ethylidene)-2H-pyran-2,6(3H)-dione (41): white solid, m.p. $143-144{ }^{\circ} \mathrm{C}$, yield 72\%; ${ }^{1} \mathrm{H}-\mathrm{NMR}\left(500 \mathrm{MHz}, \mathrm{DMSO}-d_{6}\right) \delta 13.34(\mathrm{~s}, 1 \mathrm{H}), 7.39-7.57(\mathrm{~m}, 5 \mathrm{H}), 5.63(\mathrm{~s}, 1 \mathrm{H}), 2.51(\mathrm{~s}, 3 \mathrm{H})$, $2.43(\mathrm{~s}, 3 \mathrm{H}) ;{ }^{13} \mathrm{C}-\mathrm{NMR}\left(125 \mathrm{MHz}, \mathrm{DMSO}-d_{6}\right) \delta 170.6,167.0,160.8,159.0,137.3,130.5,128.8,126.8,105.6$, 96.2, 25.5, 21.8. HRMS (ESI) calcd. for $\mathrm{C}_{14} \mathrm{H}_{13} \mathrm{NNaO}_{3}[\mathrm{M}+\mathrm{Na}]^{+}$266.0788, found 266.0792. 
(Z)-3-(1-((4-Fluorophenyl)amino)ethylidene)-4-methyl-2H-pyran-2,6(3H)-dione (4m): white solid, m.p. 137-139 ${ }^{\circ} \mathrm{C}$, yield 50\%; ${ }^{1} \mathrm{H}-\mathrm{NMR}\left(500 \mathrm{MHz}\right.$, DMSO- $\left.d_{6}\right) \delta 13.22(\mathrm{~s}, 1 \mathrm{H}), 7.36-7.47(\mathrm{~m}, 4 \mathrm{H}), 5.63(\mathrm{~s}$, $1 \mathrm{H}), 2.46(\mathrm{~s}, 3 \mathrm{H}), 2.42(\mathrm{~s}, 3 \mathrm{H}) ;{ }^{13} \mathrm{C}-\mathrm{NMR}\left(125 \mathrm{MHz}, \mathrm{DMSO}-d_{6}\right) \delta 171.0,167.0,161.0,159.2,152.4\left(\mathrm{~d}, J_{\mathrm{C}-\mathrm{F}}\right.$ $=254.2 \mathrm{~Hz}), 133.7\left(\mathrm{~d}, J_{\mathrm{C}-\mathrm{F}}=3.4 \mathrm{~Hz}\right), 129.2\left(\mathrm{~d}, J_{\mathrm{C}-\mathrm{F}}=8.7 \mathrm{~Hz}\right), 117.4\left(\mathrm{~d}, J_{\mathrm{C}-\mathrm{F}}=23.4 \mathrm{~Hz}\right), 105.7,96.3,25.6$, 21.9. HRMS (ESI) calcd. for $\mathrm{C}_{14} \mathrm{H}_{12} \mathrm{FNNaO}_{3}[\mathrm{M}+\mathrm{Na}]^{+}$284.0693, found 284.0695.

(Z)-3-(1-((4-Bromophenyl)amino)ethylidene)-4-methyl-2H-pyran-2,6(3H)-dione (4n): white solid, m.p. 161-164 ${ }^{\circ} \mathrm{C}$, yield 53\%; ${ }^{1} \mathrm{H}-\mathrm{NMR}\left(500 \mathrm{MHz}, \mathrm{DMSO}-d_{6}\right) \delta 13.21(\mathrm{~s}, 1 \mathrm{H}), 7.74(\mathrm{~d}, J=8.6 \mathrm{~Hz}, 2 \mathrm{H})$, $7.36(\mathrm{~d}, J=8.6 \mathrm{~Hz}, 2 \mathrm{H}), 5.65(\mathrm{~s}, 1 \mathrm{H}), 2.49(\mathrm{~s}, 3 \mathrm{H}), 2.42(\mathrm{~s}, 3 \mathrm{H}) ;{ }^{13} \mathrm{C}-\mathrm{NMR}\left(125 \mathrm{MHz}\right.$, DMSO- $\left.d_{6}\right) \delta$ 170.4, 166.9, 160.8, 158.9, 136.8, 133.3, 129.0, 121.6, 105.9, 96.6, 25.5, 21.9. HRMS (ESI) calcd. for $\mathrm{C}_{14} \mathrm{H}_{12} \mathrm{BrNNaO}_{3}[\mathrm{M}+\mathrm{Na}]^{+}$343.9893, found 343.9897.

(Z)-3-(1-((3,5-dichlorophenyl)amino)ethylidene)-4-methyl-2H-pyran-2,6(3H)-dione (4o): white solid, m.p. 166-167 ${ }^{\circ} \mathrm{C}$, yield $41 \%$; ${ }^{1} \mathrm{H}-\mathrm{NMR}\left(500 \mathrm{MHz}\right.$, DMSO- $\left.d_{6}\right) \delta 13.11(\mathrm{~s}, 1 \mathrm{H}), 7.58-7.70(\mathrm{~m}, 3 \mathrm{H}), 5.68(\mathrm{~s}, 1 \mathrm{H})$, $2.51(\mathrm{~s}, 3 \mathrm{H}), 2.42(\mathrm{~s}, 3 \mathrm{H}) ;{ }^{13} \mathrm{C}-\mathrm{NMR}\left(125 \mathrm{MHz}, \mathrm{DMSO}-d_{6}\right) \delta 170.4,166.7,160.7,158.7,139.9,135.4,128.3$, 126.1, 106.5, 97.1, 25.5, 22.2. HRMS (ESI) calcd. for $\mathrm{C}_{14} \mathrm{H}_{11} \mathrm{Cl}_{2} \mathrm{NNaO}_{3}[\mathrm{M}+\mathrm{Na}]^{+} 334.0008$, found 334.0012 .

(Z)-3-(1-((4-Chloro-3-(trifluoromethyl)phenyl)amino)ethylidene)-4-methyl-2H-pyran-2,6(3H)-dione (4p): white solid, m.p. $109-111^{\circ} \mathrm{C}$, yield $39 \% ;{ }^{1} \mathrm{H}-\mathrm{NMR}\left(500 \mathrm{MHz}\right.$, DMSO- $\left.d_{6}\right) \delta 13.14(\mathrm{~s}, 1 \mathrm{H}), 7.91(\mathrm{~s}, 1 \mathrm{H}), 7.87$ $(\mathrm{d}, J=8.6 \mathrm{~Hz}, 2 \mathrm{H}), 7.73(\mathrm{~d}, J=8.6 \mathrm{~Hz}, 2 \mathrm{H}), 5.67(\mathrm{~s}, 1 \mathrm{H}), 2.50(\mathrm{~s}, 3 \mathrm{H}), 2.43(\mathrm{~s}, 3 \mathrm{H}) ;{ }^{13} \mathrm{C}-\mathrm{NMR}(125 \mathrm{MHz}$, DMSO- $\left.d_{6}\right) \delta 170.1,166.5,160.5,158.3,136.9,133.4,132.4,130.3,128.2\left(\mathrm{q}, J_{\mathrm{C}-\mathrm{F}}=30.9 \mathrm{~Hz}\right), 126.5\left(\mathrm{q}, J_{\mathrm{C}-\mathrm{F}}=\right.$ $5.3 \mathrm{~Hz}), 123.2\left(\mathrm{q}, J_{\mathrm{C}-\mathrm{F}}=274.2 \mathrm{~Hz}\right), 106.4,96.9,25.2,21.8$. HRMS (ESI) calcd. for $\mathrm{C}_{15} \mathrm{H}_{11} \mathrm{ClF}_{3} \mathrm{NNaO}_{3}[\mathrm{M}$ $+\mathrm{Na}]^{+} 368.0272$, found 368.0272 .

(Z)-4-Methyl-3-(1-(p-tolylamino)ethylidene)-2H-pyran-2,6(3H)-dione (4q): white solid, m.p. $127-129{ }^{\circ} \mathrm{C}$, yield 75\%; ${ }^{1} \mathrm{H}-\mathrm{NMR}\left(500 \mathrm{MHz}\right.$, DMSO- $\left.d_{6}\right) \delta 13.29(\mathrm{~s}, 1 \mathrm{H}), 7.35(\mathrm{~d}, J=8.0 \mathrm{~Hz}, 3 \mathrm{H}), 7.27(\mathrm{~d}, J=8.0 \mathrm{~Hz}$, $3 \mathrm{H}), 5.62(\mathrm{~s}, 1 \mathrm{H}), 2.49(\mathrm{~s}, 3 \mathrm{H}), 2.42(\mathrm{~s}, 3 \mathrm{H}), 2.39(\mathrm{~s}, 3 \mathrm{H}) ;{ }^{13} \mathrm{C}-\mathrm{NMR}\left(125 \mathrm{MHz}, \mathrm{DMSO}-d_{6}\right) \delta 170.6,167.0$, $160.9,159.0,138.4,134.7,130.9,126.6,105.5,96.1,25.5,21.8,21.5$. HRMS (ESI) calcd. for $\mathrm{C}_{15} \mathrm{H}_{15} \mathrm{NNaO}_{3}$ $[\mathrm{M}+\mathrm{Na}]^{+} 280.0944$, found 280.0946 .

(Z)-3-(1-((4-Isopropylphenyl)amino)ethylidene)-4-methyl-2H-pyran-2,6(3H)-dione (4r): white solid, m.p. 134-136 ${ }^{\circ} \mathrm{C}$, yield 70\%; ${ }^{1} \mathrm{H}-\mathrm{NMR}\left(500 \mathrm{MHz}\right.$, DMSO- $\left.d_{6}\right) \delta 13.30(\mathrm{~s}, 1 \mathrm{H}), 7.42(\mathrm{~d}, J=8.0 \mathrm{~Hz}, 3 \mathrm{H}), 7.30$ $(\mathrm{d}, J=8.0 \mathrm{~Hz}, 3 \mathrm{H}), 5.62(\mathrm{~s}, 1 \mathrm{H}), 2.96-3.01(\mathrm{~m}, 1 \mathrm{H}), 2.50(\mathrm{~s}, 3 \mathrm{H}), 2.42(\mathrm{~s}, 3 \mathrm{H}), 1.24(\mathrm{~d}, J=6.9 \mathrm{~Hz}, 6 \mathrm{H})$; ${ }^{13} \mathrm{C}-\mathrm{NMR}\left(125 \mathrm{MHz}\right.$, DMSO- $\left.d_{6}\right) \delta 170.6,167.0,160.9,159.0,149.1,135.0,128.3,126.6,105.4,96.1,33.9$, 25.5, 24.6, 21.8. HRMS (ESI) calcd. for $\mathrm{C}_{17} \mathrm{H}_{19} \mathrm{NNaO}_{3}[\mathrm{M}+\mathrm{Na}]^{+} 308.1257$, found 308.1257.

(Z)-3-(1-((4-Methoxyphenyl)amino)ethylidene)-4-methyl-2H-pyran-2,6(3H)-dione (4s): white solid, m.p. 148-150 ${ }^{\circ} \mathrm{C}$, yield 71\%; ${ }^{1} \mathrm{H}-\mathrm{NMR}\left(500 \mathrm{MHz}\right.$, DMSO- $\left.d_{6}\right) \delta 13.22(\mathrm{~s}, 1 \mathrm{H}), 7.32(\mathrm{~d}, J=8.0 \mathrm{~Hz}, 3 \mathrm{H}), 7.09(\mathrm{~d}$, $J=8.0 \mathrm{~Hz}, 3 \mathrm{H}), 5.60(\mathrm{~s}, 1 \mathrm{H}), 3.84(\mathrm{~s}, 3 \mathrm{H}), 2.47(\mathrm{~s}, 3 \mathrm{H}), 2.42(\mathrm{~s}, 3 \mathrm{H}) ;{ }^{13} \mathrm{C}-\mathrm{NMR}\left(125 \mathrm{MHz}\right.$, DMSO- $\left.d_{6}\right) \delta$ $170.8,167.0,160.9,159.6,159.0,129.9,128.1,115.6,105.3,95.9,56.4,25.5,21.7$. HRMS (ESI) calcd. for $\mathrm{C}_{15} \mathrm{H}_{15} \mathrm{NNaO}_{4}[\mathrm{M}+\mathrm{Na}]^{+} 296.0893$, found 296.0895.

(Z)-4-Methyl-3-(1-((4-(trifluoromethoxy)phenyl)amino)ethylidene)-2H-pyran-2,6(3H)-dione (4t): white solid, m.p. $146-147^{\circ} \mathrm{C}$, yield 57\%; ${ }^{1} \mathrm{H}-\mathrm{NMR}\left(500 \mathrm{MHz}, \mathrm{DMSO}-d_{6}\right) \delta 13.28(\mathrm{~s}, 1 \mathrm{H}), 7.55(\mathrm{~s}, 4 \mathrm{H}), 5.66(\mathrm{~s}, 1 \mathrm{H})$, $2.50(\mathrm{~s}, 3 \mathrm{H}), 2.43(\mathrm{~s}, 3 \mathrm{H}) ;{ }^{13} \mathrm{C}-\mathrm{NMR}\left(125 \mathrm{MHz}, \mathrm{DMSO}-d_{6}\right) \delta 170.6,166.9,160.8,158.9,148.2,136.5,129.0$, 123.0, $120.9\left(\mathrm{q}, J_{\mathrm{C}-\mathrm{F}}=260.7 \mathrm{~Hz}\right), 106.0,96.6,25.5,21.9$. HRMS (ESI) calcd. for $\mathrm{C}_{15} \mathrm{H}_{12} \mathrm{~F}_{3} \mathrm{NNaO}_{4}[\mathrm{M}+$ $\mathrm{Na}^{+}$350.0611, found 350.0612 .

(Z)-3-(1-(Tert-butylamino)ethylidene)-4-methyl-2H-pyran-2,6(3H)-dione (4u): white solid, m.p. $133-135{ }^{\circ} \mathrm{C}$, yield $44 \% ;{ }^{1} \mathrm{H}-\mathrm{NMR}\left(500 \mathrm{MHz}, \mathrm{DMSO}-d_{6}\right) \delta 8.18(\mathrm{~s}, 1 \mathrm{H}), 6.10(\mathrm{~s}, 1 \mathrm{H}), 2.20(\mathrm{~s}, 3 \mathrm{H}), 2.09(\mathrm{~s}, 3 \mathrm{H}), 1.35$ 
(s, 9H); ${ }^{13} \mathrm{C}-\mathrm{NMR}\left(125 \mathrm{MHz}\right.$, DMSO- $\left.d_{6}\right) \delta 163.9,160.8,158.2,154.8,118.0,110.3,50.8,28.1,18.7,17.7$. HRMS (ESI) calcd. for $\mathrm{C}_{12} \mathrm{H}_{18} \mathrm{NO}_{3}[\mathrm{M}+\mathrm{H}]^{+}$224.1281, found 224.1281.

4,6-Dimethyl-2-oxo-N-(2,4,4-trimethylpentan-2-yl)-2H-pyran-5-carboxamide (4v): white solid, m.p. 129-131 ${ }^{\circ} \mathrm{C}$, yield 47\%; ${ }^{1} \mathrm{H}-\mathrm{NMR}\left(500 \mathrm{MHz}\right.$, DMSO- $\left.d_{6}\right) \delta 7.98(\mathrm{~s}, 1 \mathrm{H}), 6.10(\mathrm{~s}, 1 \mathrm{H}), 2.23(\mathrm{~s}, 3 \mathrm{H})$, $2.12(\mathrm{~s}, 3 \mathrm{H}), 1.78(\mathrm{~s}, 2 \mathrm{H}), 1.42(\mathrm{~s}, 6 \mathrm{H}), 1.02(\mathrm{~s}, 9 \mathrm{H}) ;{ }^{13} \mathrm{C}-\mathrm{NMR}\left(125 \mathrm{MHz}, \mathrm{DMSO}-d_{6}\right) \delta 164.6,161.6,159.5$, $155.8,119.0,111.3,56.1,52.1,32.2,32.1,29.2,20.0,18.9$. HRMS (ESI) calcd. for $\mathrm{C}_{16} \mathrm{H}_{26} \mathrm{NO}_{3}[\mathrm{M}+\mathrm{H}]^{+}$ 280.1907, found 280.1909 .

4,6-Dimethyl-2-oxo-N-(o-tolyl)-2H-pyran-5-carboxamide (4w): white solid, m.p. 138-139 ${ }^{\circ} \mathrm{C}$, yield 37\%; ${ }^{1} \mathrm{H}-\mathrm{NMR}\left(500 \mathrm{MHz}, \mathrm{DMSO}-d_{6}\right) \delta 10.03(\mathrm{~s}, 1 \mathrm{H}), 7.18-7.47(\mathrm{~m}, 4 \mathrm{H}), 6.22(\mathrm{~s}, 1 \mathrm{H}), 2.37(\mathrm{~s}, 3 \mathrm{H}), 2.29$ (s, 3H), $2.23(\mathrm{~s}, 3 \mathrm{H}) ;{ }^{13} \mathrm{C}-\mathrm{NMR}(125 \mathrm{MHz}$, DMSO-d 6 ) $\delta 164.2,161.5,160.0,155.5,136.3,133.4,131.4,127.1$, 127.0, 126.5, 118.3, 111.5, 20.1, 19.1, 19.0. HRMS (ESI) calcd. for $\mathrm{C}_{15} \mathrm{H}_{16} \mathrm{NO}_{3}[\mathrm{M}+\mathrm{H}]^{+} 258.1125$, found 258.1129 .

\section{Conclusions}

In summary, owing to an unexpected reaction, a concise, operationally simple method has been developed which provides expedient access to synthesize a new type of $\beta$-enaminone-containing compound. The unique structure of isodehydracetic acid strongly suggests that the reaction mechanism involves the formation of highly reactive ketene intermediates.

Supplementary Materials: The following are available online, NMR data and computational results.

Author Contributions: Conceptualization, methodology, synthesis and characterization of compounds, writing - original draft preparation, funding acquisition, D.W.; Computation, writing-review and editing, H.S.; All authors have read and agreed to the published version of the manuscript.

Funding: This research was funded by National Natural Science Foundation of China (No. 31901909), and Ph.D. Research Startup Foundation of Shanxi Agricultural University (No. 2018YJ15).

Conflicts of Interest: The authors declare no conflict of interest.

\section{Abbreviations}

$\begin{array}{ll}\text { DCC } & N, N^{\prime} \text {-Dicyclohexylcarbodiimide } \\ \text { DMAP } & \text { 4-Dimethylaminopyridine } \\ \text { EDC } & \text { 1-(3-Dimethylaminopropyl)-3-ethylcarbodiimide hydrochloride } \\ \text { HOBT } & \text { Hydroxybenzotriazole } \\ \text { HATU } & \text { 2-(7-Azabenzotriazol-1-yl)- } N, N, N^{\prime}, N^{\prime} \text {-tetramethyluronium hexafluorophosphate } \\ \text { DIPEA } & N, N \text {-Diisopropylethylamine } \\ \text { DMF } & N, N \text {-Dimethylformamide } \\ \text { DCM } & \text { Dichloromethane } \\ \text { THF } & \text { Tetrahydrofuran }\end{array}$

\section{Appendix A}

The X-ray crystallographic data for compound 3 has been deposited at the Cambridge Crystallographic Data Centre (CCDC) under deposition number 1986602. Crystal data for compound 3, $\mathrm{C}_{12} \mathrm{H}_{17} \mathrm{NO}_{3}: M r=223.27$, orthorhombic, space group Pbca, a = 10.5426(9) $\AA, \mathrm{b}=10.0532(8) \AA, \mathrm{c}=23.136(2) \AA, V=2452.1(4) \AA^{3}, \mathrm{Z}=8$, $\rho_{\text {calcd }}=1.210 \mathrm{~g} \mathrm{~cm}^{3}, \mu=1.479 \mathrm{~mm}^{-1}, F(000)=960$, crystal size $=0.43 \times 0.40 \times 0.18 \mathrm{~mm}^{3}$. A total of 11,287 reflections were collected of which 2163 reflections were unique $(R(\mathrm{int})=0.0625) . \mathrm{R}_{1}=0.0584$ for 2163 independent reflections with $[I>2 \sigma(I)], \mathrm{w} R_{2}=0.1446$.

\section{References}

1. Sollenberger, P.Y.; Martin, R.B. Mechanism of Enamine Hydrolysis. J. Am. Chem. Soc. 1970, 92, 4261-4270. [CrossRef] 
2. Blau, K.; Burgemeister, I.; Grasnick, J.; Voerckel, V. Studies on the oxidation of enamines with molecular oxygen. III. Oxidation of some amino Styrenes. J. Für Prakt. Chem. 1991, 333, 455-466. [CrossRef]

3. Tan, W.; Du, B.-X.; Li, X.; Zhu, X.; Shi, F.; Tu, S.-J. Catalytic Asymmetric Aza-ene reaction of 3-indolylmethanols with cyclic enaminones: Enantioselective approach to C3-functionalized indoles. J. Org. Chem. 2014, 79, 4635-4643. [CrossRef]

4. Gayon, E.; Szymczyk, M.; Gérard, H.; Vrancken, E.; Campagne, J.-M. Stereoselective and catalytic access to ß-enaminones: An entry to pyrimidines. J. Org. Chem. 2012, 77, 9205-9220. [CrossRef] [PubMed]

5. Turunen, B.J.; Georg, G.I. Amino acid-derived enaminones: A study in ring formation providing valuable asymmetric synthons. J. Am. Chem. Soc. 2006, 128, 8702-8703. [CrossRef] [PubMed]

6. Popov, S.A.; Gatilov, Y.V.; Rybalova, T.V.; Tkachev, A.V. Study of chiral $\beta$-enaminones prepared from pyrrolidine, cytisine, salsoline and 2-Amino-1-(4-nitrophenyl)propane-1,3-diol: Resolution of salsoline via Diastereomeric Modified Carane-Type $\beta$-Enaminones. Tetrahedron Asymmetry 2003, 14, 233-238. [CrossRef]

7. Carson, J.R.; Carmosin, R.J.; Pitis, P.M.; Vaught, J.L.; Almond, H.R.; Stables, J.P.; Wolf, H.H.; Swinyard, E.A.; White, H.S. Aroyl(aminoacyl)pyrroles, a new class of anticonvulsant agents. J. Med. Chem. 1997, 40, 1578-1584. [CrossRef] [PubMed]

8. Edafiogho, I.O.; Hinko, C.N.; Chang, H.; Moore, J.A.; Mulzac, D.; Nicholson, J.M.; Scott, K.R. Synthesis and anticonvulsant activity of enaminones. J. Med. Chem. 1992, 35, 2798-2805. [CrossRef] [PubMed]

9. Scott, K.R.; Rankin, G.O.; Stables, J.P.; Alexander, M.S.; Edafiogho, I.O.; Farrar, V.A.; Kolen, K.R.; Moore, J.A.; Sims, L.D.; Tonnu, A.D. Synthesis and anticonvulsant activity of enaminones. 3. Investigations on 4'-, 3'-, and 2'-Substituted and polysubstituted anilino compounds, sodium channel binding studies, and toxicity evaluations1,2. J. Med. Chem. 1995, 38, 4033-4043. [CrossRef]

10. Faghih, R.; Gopalakrishnan, M.; Briggs, C.A. Allosteric Modulators of the $\alpha 7$ nicotinic acetylcholine receptor. J. Med. Chem. 2008, 51, 701-712. [CrossRef]

11. Hogenkamp, D.J.; Johnstone, T.B.C.; Huang, J.-C.; Li, W.-Y.; Tran, M.; Whittemore, E.R.; Bagnera, R.E.; Gee, K.W. Enaminone amides as novel orally active GABAA receptor modulators. J. Med. Chem. 2007, 50, 3369-3379. [CrossRef] [PubMed]

12. Naringrekar, V.H.; Stella, V.J. Mechanism of Hydrolysis and structure-stability relationship of enaminones as potential prodrugs of model primary amines. J. Pharm. Sci. 1990, 79, 138-146. [CrossRef] [PubMed]

13. Lue, P.; Greenhill, J.V. Enaminones in heterocyclic synthesis. In Advances in Heterocyclic Chemistry; Katritzky, A.R., Ed.; Academic Press, Inc.: San Diego, CA, USA, 1996; Volume 67, pp. 207-343.

14. Kascheres, C.M. The chemistry of enaminones, diazocarbonyls and small rings: Our contribution. J. Brazil. Chem. Soc. 2003, 14, 945-969. [CrossRef]

15. Huma, R.; Mahmud, T.; Idrees, N.; Saif, M.J.; Munir, R.; Akbar, N. Crystal structure and quantum chemical studies of a novel push-pull enaminone: 3-Chloro-4-((4-bromophenyl)amino)pent-3-en-2-one. J. Chem. Crystallogr. 2019. [CrossRef]

16. Alnajjar, A.-A.; Abdelkhalik, M.M.; Al-Enezi, A.; Elnagdi, M.H. Enaminones as Building blocks in heterocyclic syntheses: Reinvestigating the product structures of enaminones with malononitrile. A Novel Route to 6-Substituted-3-Oxo-2,3-Dihydropyridazine-4-Carboxylic acids. Molecules 2009, 14, 68-77. [CrossRef]

17. Niphakis, M.J.; Turunen, B.J.; Georg, G.I. Synthesis of 6- and 7-Membered cyclic enaminones: Scope and mechanism. J. Org. Chem. 2010, 75, 6793-6805. [CrossRef]

18. Chattopadhyay, A.K.; Hanessian, S. Cyclic enaminones. Part II: Applications as versatile intermediates in alkaloid synthesis. Chem. Commun. 2015, 51, 16450-16467. [CrossRef]

19. Chattopadhyay, A.K.; Hanessian, S. Cyclic enaminones. Part I: Stereocontrolled synthesis using diastereoselective and catalytic asymmetric methods. Chem. Commun. 2015, 51, 16437-16449. [CrossRef]

20. Yi, S.; Varun, B.V.; Choi, Y.; Park, S.B. A brief overview of two major strategies in diversity-oriented synthesis: Build/couple/pair and ring-distortion. Front. Chem. 2018, 6, 507. [CrossRef]

21. O' Connor, C.J.; Beckmann, H.S.G.; Spring, D.R. Diversity-oriented synthesis: Producing chemical tools for dissecting biology. Chem. Soc. Rev. 2012, 41, 4444-4456. [CrossRef]

22. Pavlinov, I.; Gerlach, E.M.; Aldrich, L.N. Next generation diversity-oriented synthesis: A paradigm shift from chemical diversity to biological diversity. Org. Biomol. Chem. 2019, 17, 1608-1623. [CrossRef] [PubMed]

23. Cho, B.R.; Jeong, H.C.; Seung, Y.J.; Pyun, S.Y. Ketene-forming eliminations from aryl phenylacetates promoted by $\mathrm{R}_{2} \mathrm{NH} / \mathrm{R}_{2} \mathrm{NH}_{2}{ }^{+}$in Aqueous MeCN. Mechanistic borderline between E2 and E1cb. J. Org. Chem. 2002, 67, 5232-5238. [CrossRef] [PubMed] 
24. Nahmany, M.; Melman, A. Facile Acylation of sterically hindered alcohols through ketene intermediates. Org. Lett. 2001, 3, 3733-3735. [CrossRef] [PubMed]

25. van der Steen, F.H.; van Koten, G. Syntheses of 3-amino-2-azetidinones: A literature survey. Tetrahedron 1991, 47, 7503-7524. [CrossRef]

26. Deketelaere, S.; Van Nguyen, T.; Stevens, C.V.; D'Hooghe, M. Synthetic approaches toward monocyclic 3-Amino- $\beta$-lactams. ChemistryOpen 2017, 6, 301-319. [CrossRef]

27. Smith, N.R.; Wiley, R.H. Isodehydroacetic acid and ethyl isodehydroacetate. Organ. Synth. $2003,76$. [CrossRef]

Sample Availability: Samples of the compounds are available from the authors.

(C) 2020 by the authors. Licensee MDPI, Basel, Switzerland. This article is an open access article distributed under the terms and conditions of the Creative Commons Attribution (CC BY) license (http://creativecommons.org/licenses/by/4.0/). 